\title{
Clinical recommendations for the use of lapatinib ditosylate plus capecitabine for patients with advanced or metastatic HER2-positive breast cancer
}

\author{
Raymond D SNYDER, ${ }^{1}$ Frances M BOYLE, ${ }^{2}$ Arlene CHAN, ${ }^{3}$ Paul S CRAFT, ${ }^{4}$ \\ Richard DE BOER, ${ }^{5}$ Paul N MAINWARING, ${ }^{6}$ Nicole J McCARTHY ${ }^{7}$ and \\ Nicholas R WILCKEN ${ }^{8}$ \\ ${ }^{1}$ St Vincent's Hospital, Melbourne, Victoria, ${ }^{2}$ Patricia Ritchie Centre, Mater Hospital, Sydney, New South Wales, ${ }^{3}$ Mount \\ Hospital, Perth, Western Australia, ${ }^{4}$ Canberra Hospital, Canberra, Australian Capital Territory, ${ }^{5}$ Royal Melbourne Hospital, \\ Melbourne, Victoria, ${ }^{6}$ Mater Adult Hospital, Brisbane, Queensland, ${ }^{7}$ Royal Brisbane and Women's Hospital, Brisbane, \\ Queensland and ${ }^{8}$ Westmead Hospital, Westmead, Sydney, New South Wales, Australia
}

\begin{abstract}
Primary and acquired resistance to trastuzumab pose a therapeutic challenge when treating patients with HER2 (erbB-2)-positive locally advanced or metastatic breast cancer (MBC). The recent introduction of lapatinib (Tykerb/Tyverb, GlaxoSmithKline, Brentford, UK) provides a new management option for such patients. A prospective, randomized phase III clinical trial has confirmed that lapatinib in combination with capecitabine extends time to progressive disease in HER2-positive MBC, compared with capecitabine alone in patients with disease progression despite prior anthracycline, taxane and trastuzumab therapy. Preliminary data also indicate that lapatinib may exert a beneficial effect on brain metastases, a common sanctuary site for HER2-positive breast cancer following trastuzumab treatment. The tolerability of lapatinib is commensurate with that of other erbB family tyrosine kinase inhibitors and no significant new adverse events have emerged following its introduction into clinical practice. In particular, no additive cardiotoxicity has been observed when lapatinib is prescribed after trastuzumab therapy. Based on the published literature and supplemented by clinical experience, this article provides practical management recommendations for the use of lapatinib plus capecitabine in patients with MBC. Issues addressed include patient selection, baseline evaluation and monitoring for clinical benefit. The minimization and management of adverse events is also discussed in detail, particularly the dermatological and gastrointestinal effects, which are the most clinically significant side-effects of lapatinib therapy. Further recommendations cover the minimization of drug interactions, anticipated dosing alterations and the optimal employment of oral anticancer regimens.
\end{abstract}

Key words: breast neoplasm, epidermal growth factor receptor (EGFR), erbB-2 receptor (HER2), lapatinib, medication therapy management.

\section{INTRODUCTION}

The addition of trastuzumab to cytotoxic chemotherapy regimens can prolong median survival in patients with

Correspondence: Dr Raymond Snyder, Department of Medical Oncology, St Vincent's Hospital, Vic. 3065, Australia. Email: raymond.snyder@svhm.org.au

Accepted for publication 6 January 2009.

(C) 2009 The Authors

Journal Compilation @ C Blackwell Publishing Asia Pty Ltd
erbB-2 (HER2)-overexpressing locally advanced or metastatic breast cancer (MBC). ${ }^{1-3}$ However, primary resistance to trastuzumab has been reported in $12-49 \%$ of patients receiving this agent in combination with chemotherapy, and occurring even more frequently during monotherapy. ${ }^{1,2,4}$ It is also apparent that acquired resistance can develop within 3-7 months of commencing trastuzumab. ${ }^{1,4}$ Until recently, clinicians have had few evidence-based alternatives for treating $\mathrm{MBC}$ after 
progression following anthracycline, taxane and trastuzumab therapy. Lapatinib ditosylate (GW572016, Tykerb/Tyverb, GlaxoSmithKline, Brentford, UK) is a new therapeutic option in this setting. This article outlines the optimal clinical use of lapatinib in combination with capecitabine for patients with trastuzumabresistant, HER2-positive MBC.

\section{CLINICAL TRIAL RESULTS AND REGULATORY APPROVALS}

An important preclinical finding in $\mathrm{MBC}$ is that cells resistant to trastuzumab may remain sensitive to other HER2-targeted therapies such as lapatinib. ${ }^{5-10}$ A synthetic, small-molecule agent, lapatinib acts in tumor cells to inhibit the tyrosine kinase domain of both HER2 and epidermal growth factor receptors (EGFR; erbB-1). Active at nanomolar concentrations, lapatinib has been associated with retarded tumor growth in murine xenograft models and with inhibition of tumor cell proliferation in vitro, including cell lines previously exposed to trastuzumab. ${ }^{11,12}$ Data in the neoadjuvant setting suggest that lapatinib may inhibit the self-renewal of HER2-overexpressing breast cancer stem cells, rendering them more sensitive to cytotoxic chemotherapy. ${ }^{13}$

Based on positive efficacy and safety data from singleagent phase I and II clinical studies, ${ }^{14-17}$ a randomized, double-blind, comparative trial of lapatinib plus capecitabine versus capecitabine alone was initiated in patients with HER2-positive, trastuzumab-resistant MBC. ${ }^{18}$ As this trial (EGF100151) formed the basis for global regulatory approvals of lapatinib, it is pertinent to consider key inclusion and exclusion criteria. Of the women enrolled in EGF100151, 96\% had MBC and the remainder had locally advanced disease (stage IIIB or IIIC with T4 primary tumor). All the patients had progressed after one or more lines of prior treatment which included an anthracycline, a taxane and at least 6 weeks of trastuzumab in the metastatic setting. Prior exposure to capecitabine was not allowed. Although not excluded, patients with brain metastases were required to have been clinically stable for at least 3 months after completing whole-brain radiation plus anticonvulsants and steroids. Owing to concerns over the potential cardiotoxicity of HER2-directed agents, all participants had to demonstrate a left ventricular ejection fraction (LVEF) above the institution's lower limit of normal, plus adequate renal, hepatic, hematological and gastrointestinal function. ${ }^{18}$

In total, 399 patients were randomized to receive either capecitabine monotherapy $\left(2500 \mathrm{mg} / \mathrm{m}^{2}\right.$ divided across two doses daily for 14 days on a 21-day cycle), or capecitabine $\left(2000 \mathrm{mg} / \mathrm{m}^{2}\right.$ on the same regimen) plus lapatinib (1250 mg once daily on a continuous basis). ${ }^{19}$ EGF100151 was originally designed for $90 \%$ power to detect a $50 \%$ difference in time to progression (TTP) and $80 \%$ power to detect a $30 \%$ difference in median overall survival. ${ }^{18}$ However, the trial was stopped at the first planned interim analysis when the lapatinib combination regimen demonstrated superiority over capecitabine monotherapy for TTP, the primary outcome measure. ${ }^{18}$ Subsequent analysis confirmed the significant extension of TTP and progression-free survival (PFS) with the lapatinib plus capecitabine combination, although mature overall survival data are not yet available (Table 1). ${ }^{19}$

Following this positive outcome, in March 2007 the US Food and Drug Administration approved lapatinib in combination with capecitabine for HER2-positive advanced or $\mathrm{MBC}$ after prior anthracycline, taxane and trastuzumab therapy. ${ }^{20}$ In July 2007 lapatinib was also approved in Australia, and it is now governmentsubsidized for patients with metastatic disease after progression during trastuzumab treatment. ${ }^{21,22}$ Many other Asia-Pacific nations have approved lapatinib for use in similar settings, including Hong Kong, India, Indonesia, Macau, Malaysia, New Zealand, the Philippines, Singapore and South Korea. In June 2008 the European Medicines Agency also granted marketing authorization, conditional upon updated survival data from EGF100151 and the instigation of a phase III trial comparing the impact of lapatinib- or trastuzumab-based regimens on the central nervous system (CNS) as a relapse site in HER2-positive MBC. ${ }^{23}$ The clinical role of lapatinib is now also being recognized in national breast cancer management guidelines. ${ }^{24}$

\section{PATIENT SELECTION}

Patients who qualify for lapatinib plus capecitabine may be quite heterogeneous, particularly regarding the number and composition of previous treatment regimens. However, the fundamental criterion for initiating lapatinib therapy is documented progression of HER2positive locally advanced or MBC despite treatment with trastuzumab.

HER2 protein overexpression is the only biomarker that has been demonstrated to predict clinical response to lapatinib in breast cancer. ${ }^{12,15,16,19,25}$ No significant association has been identified between PFS and HER2 extracellular domain (ECD) levels, baseline EGFR or EGFR ECD. ${ }^{15,19}$ While retesting for HER2 overexpres- 
Table 1 Results from EGF100151, a phase III multicentre, blinded, comparative trial comparing lapatinib plus capecitabine to capecitabine alone in patients with trastuzumab-resistant, HER2-positive advanced or metastatic breast cancer

\begin{tabular}{|c|c|c|c|}
\hline Outcome measure & $\begin{array}{l}\text { Lapatinib } \\
\text { plus capecitabine }\end{array}$ & $\begin{array}{l}\text { Capecitabine } \\
\text { monotherapy }\end{array}$ & $\begin{array}{l}\text { Statistical } \\
\text { significance }\end{array}$ \\
\hline $\begin{array}{l}\text { Median time to progression } \\
\text { - Initial analysis (months) } \\
\text { - Follow-up analysis (months) }\end{array}$ & $\begin{array}{l}8.4 \\
6.2\end{array}$ & $\begin{array}{l}4.4 \\
4.3\end{array}$ & $\begin{array}{l}P<0.001 \\
P<0.001\end{array}$ \\
\hline $\begin{array}{l}\text { Median progression-free survival } \\
\text { - Initial analysis (months) }\end{array}$ & 8.4 & 4.1 & $P<0.001$ \\
\hline $\begin{array}{l}\text { Overall response rate } \\
\text { - Initial analysis }(\%) \\
\text { - Follow-up analysis }(\%)\end{array}$ & $\begin{array}{l}22 \\
24\end{array}$ & $\begin{array}{l}14 \\
14\end{array}$ & $\begin{array}{l}P=0.09 \\
P=0.017\end{array}$ \\
\hline $\begin{array}{l}\text { Clinical benefit rate } \\
\text { - Initial analysis }(\%) \\
\text { - Follow-up analysis ( } \%)\end{array}$ & $\begin{array}{l}27 \\
29\end{array}$ & $\begin{array}{l}18 \\
17\end{array}$ & $P=\overline{-} .008$ \\
\hline
\end{tabular}

Where available, results from both the initial data censure $(n=324)^{18}$ and follow-up analysis $(n=399)^{19}$ are presented. ${ }^{\dagger}$ Proportion of patients achieving complete or partial objective tumor response. ^Proportion of patients achieving complete response, partial response or stable disease lasting $\geq 6$ months.

sion is unnecessary prior to commencing lapatinib, HER2-negative primary tumors have been known to transform to HER2 positivity and the HER2 status of disseminated cells differs from the primary tumor in $\geq 10 \%$ of MBC cases. ${ }^{26,27}$ It may therefore be prudent to test any subsequent biopsies for HER2 and hormone receptor status. Although in situ hybridization (ISH) offers the most robust means to assess HER2 overexpression, ${ }^{28}$ this technique is currently more expensive than immunohistochemistry (IHC). Therefore, IHC represents a reasonable first stage for HER2 testing, but equivocal results should be supplemented by a validated ISH technique. Despite ongoing investigation of other biomarkers for lapatinib sensitivity, ${ }^{29}$ no other assessment of tumor characteristics is currently recommended.

Patient selection should reflect the entry criteria for the pivotal trial. Approximately half of the patients in EGF100151 were hormone receptor negative, meaning that endocrine receptor status should not alter the decision to use lapatinib. ${ }^{18}$ An unpublished analysis shows that about $80 \%$ of trial patients had received only one or two prior lines of chemotherapy with trastuzumab for metastatic disease (data on file, GlaxoSmithKline). The median duration of prior trastuzumab treatment was approximately 43 weeks, with subgroup analysis suggesting that response to lapatinib plus capecitabine was unaffected by time since trastuzumab cessation. ${ }^{18}$ While some patients with Eastern Cooperative Oncology Group (ECOG) performance status 2 may remain fit enough for lapatinib plus capecitabine therapy, care should be exercised as EGF100151 was restricted to
ECOG 0-1 patients, although most had three or more disease sites. ${ }^{18}$ As the CNS provides a common sanctuary site for trastuzumab-resistant $\mathrm{MBC},{ }^{30,31}$ preclinical models and EGF100151 subgroups have both been evaluated to assess the effect of lapatinib regimens on brain metastases. ${ }^{19,32}$ The encouraging preliminary results were echoed by several prospective and observational studies of lapatinib for brain metastases in MBC, but firm recommendations await confirmation via an ongoing phase III trial. ${ }^{33-36}$ At present, patients with brain metastases remain eligible for lapatinib therapy, even after prior cranial irradiation.

When considering a patient for lapatinib plus capecitabine therapy, attention should be paid to the known contraindications and precautions associated with capecitabine. Assessments specifically required prior to the initiation of lapatinib are shown in Table 2. Patients were required to display adequate baseline hepatic function in both the key phase I study of lapatinib and in EGF100151, and no serious derangements of liver function were reported in either. ${ }^{17-19}$ However, pharmacokinetic studies in patients with moderate (Child-Pugh score $7-9)$ or severe (>9) hepatic impairment demonstrated $56 \%$ and $85 \%$ increases in systemic exposure, respectively, compared to patients with normal hepatic function. ${ }^{37}$ Therefore, clinicians should be cautious when initiating lapatinib plus capecitabine in patients with marked hepatic impairment, for instance, alanine or aspartate aminotransferase (ALT/AST) levels $\geq$ thrice the upper limit of normal or baseline total bilirubin $>2 \mathrm{mg} / \mathrm{dL}(34 \mu \mathrm{mol} / \mathrm{L}) .{ }^{17}$ 
Table 2 Recommended monitoring schedule before and during lapatinib treatment. The appearance of specific adverse events such as a decline in left ventricular ejection fraction (LVEF) or elevated, liver function tests (LFT) may necessitate more frequent monitoring

\begin{tabular}{|c|c|c|}
\hline Timing & Evaluation & Criteria \\
\hline \multirow[t]{5}{*}{ Baseline } & Tumor biology & HER2-positive primary tumor $(\mathrm{ICH} 3+\text { or FISH ratio }>2.2)^{\dagger}$ \\
\hline & LVEF & Greater than institutional lower limit of normal ${ }^{\ddagger}$ \\
\hline & LFT & $\begin{array}{l}\text { Within institutional range for upper limits of normal - caution in } \\
\text { patients with moderate to severe hepatic impairment } \\
\S\end{array}$ \\
\hline & Bowel frequency & Document patient's normal range \\
\hline & Concurrent medication & Check for potential CYP3A4 interactions \\
\hline \multirow[t]{2}{*}{1 week } & Bowel frequency & Review presence/absence of early onset diarrhea \\
\hline & Symptom history & Review for skin effects, pulmonary symptoms or other events \\
\hline \multirow[t]{2}{*}{ Each visit (4-6 weekly) } & LFT & Monitor for changes monthly, or as clinically indicated \\
\hline & Symptom history & Review for diarrhea, skin effects or other adverse events \\
\hline 3 monthly & LVEF & Greater than institutional lower limit of normal ${ }^{\ddagger}$ \\
\hline \multirow{3}{*}{$\begin{array}{l}\text { Subsequent monitoring } \\
\text { as clinically indicated }\end{array}$} & LVEF & Greater than institutional lower limit of normal ${ }^{\ddagger}$ \\
\hline & LFT & Within institutional range for upper limits of normal \\
\hline & Symptom history & Review for diarrhea, skin effects or other adverse events \\
\hline
\end{tabular}

Clinicians should review patients at least once every month. Consult local product information for suggested capecitabine assessments. ${ }^{\dagger}$ Assessments undertaken prior to initiation of trastuzumab are acceptable. ${ }^{\ddagger}$ In Australia, reimbursement criteria for lapatinib require baseline LVEF $\geq 45 \% .{ }^{22}$

${ }^{\S}$ Refer to local product information for specific requirements.

Concerns regarding potential cardiotoxicity with HER2-directed therapy meant that patients in EGF100151 required normal cardiac function at entry and regular LVEF monitoring. ${ }^{38-40}$ Each patient's cardiac function should therefore be assessed at baseline to ensure that it falls within institutional limits prior to commencing lapatinib. While MBC patients may present with reduced LVEF, a recent pooled analysis of over 3000 patients found that prior anthracycline or trastuzumab treatment was not predictive of adverse cardiac events while receiving lapatinib for solid tumors. ${ }^{38}$ As there are currently no data evaluating the safety of lapatinib in patients with compromised baseline LVEF, the potential clinical benefit should be weighed against the risk of cardiotoxicity, perhaps in consultation with a cardiologist. ${ }^{39}$

\section{INITIATION OF THERAPY}

Cancer treatment with oral agents such as lapatinib requires attention to patient education in order to optimize adherence and to minimize the risk of adverse events. ${ }^{41,42}$ Treatment should ideally be initiated by an experienced medical oncologist working within a multidisciplinary team that includes dedicated oncology nurses and pharmacists. Wherever possible, the responsible physician should discuss treatment requirements, expectations and warnings directly with the patient (Table 3). All advice should be reinforced by nursing and pharmacy staff and supplemented with written information..$^{41,42}$ The possibility of patient confusion about dosing should be investigated in cases of apparent inefficacy or unanticipated toxicity; education is similarly important for patients with CNS involvement who may exhibit cognitive impairment. Given the complexity of the dosing regimen, the use of patient diaries or dosing calendars is encouraged to assist with adherence (Appendix 1). ${ }^{41}$ Lapatinib tablets should not be removed from their foil packets prior to ingestion, precluding the use of Webster packs. However, medication trays can be cut into strips of five $250 \mathrm{mg}$ tablets to prepare a $1250 \mathrm{mg}$ daily dose.

In general the dosing of lapatinib and capecitabine should follow local approved product information documents. Many additional agents can be utilized during therapy - for instance, bisphosphonates to control bone pain - but the possibility of drug interactions may necessitate dose adjustment (Table 4). ${ }^{37,43,44}$ As a class the oral tyrosine kinase inhibitors interact with cytochrome P450 enzymes, particularly CYP3A4, and where possible clinicians should aim to revise or replace the interacting agent rather than amending lapatinib or capecitabine. This strategy is particularly relevant for antihypertensives, anticonvulsants and steroids, plus grapefruit and its juice or complementary therapies such as St John's wort. ${ }^{37}$ Treatments that alter gastric motility or absorption may also affect systemic exposure to oral lapatinib, as can severe or prolonged emesis, diarrhea or 
Table 3 A suggested list of issues to discuss with patients prior to commencing lapatinib plus capecitabine therapy

\begin{tabular}{ll}
\hline Issue & Comments \\
\hline $\begin{array}{l}\text { Lapatinib dosing } \\
\text { Capecitabine dosing }\end{array}$ & $\begin{array}{l}\text { Continuous therapy, once daily without food } \\
\text { Tisk of diarrhea daily with food for 14 days followed by a 7-day break } \\
\text { Explain baseline bowel frequency; definition of serious diarrhea and when to contact the oncologist or } \\
\text { hospital; management including medication and dietary changes } \\
\text { Outline protective strategies against skin trauma; definition of serious events and when to contact the } \\
\text { oncologist or hospital; symptomatic treatments such as emollients }\end{array}$ \\
$\begin{array}{l}\text { Explain main symptoms of heart failure or interstitial lung disease, including when to contact the } \\
\text { lung function } \\
\text { Drug interactions }\end{array}$ & $\begin{array}{l}\text { Tell patient to inform the oncologist before commencing any new treatments, including } \\
\text { complementary therapies }\end{array}$ \\
\hline
\end{tabular}

constipation. While the concurrent ingestion of lapatinib and food has been contentious, ${ }^{45-49}$ it is recommended that lapatinib should continue to be given on an empty stomach. There are no data regarding cranial irradiation concurrent with lapatinib plus capecitabine, but administration of capecitabine is generally not recommended during radiotherapy.

Prior to commencing treatment with lapatinib plus capecitabine, patients should be given clear advice that diarrhea may become serious and what to do if it develops. It may be advisable to provide patients with a course of antidiarrheal agent such as loperamide at the outset of treatment, or at least ensure that patients or carers can readily obtain antidiarrheals if required. Patients should be instructed to inform their doctor if severe diarrhea develops - even if antidiarrheal therapy appears effective - as dose delay or discontinuation may be warranted. ${ }^{37}$

\section{ASSESSING CLINICAL BENEFIT}

Once treatment has begun, a range of measures can assist both clinicians and patients to determine the level of benefit derived from lapatinib and capecitabine. Mature overall survival data remain to be published for EGF100151, but median TTP was significantly extended in the combination arm versus capecitabine alone (Table 1). ${ }^{19}$ A significant improvement in clinical benefit rate (comprising complete response, partial response and stable disease lasting $\geq 6$ months) was also observed in $29 \%$ of patients taking the combination regimen, versus $17 \%$ of those on capecitabine monotherapy. ${ }^{19}$ At present, there are no published data for other objective measures of treatment outcome, such as changes in performance status, time to treatment failure or rehospitalization rates.
Assessment of treatment should consider its effect on symptoms and patient quality of life, especially in the setting of relapsed MBC. An updated analysis of EGF100151 reported that $14 \%$ of patients in each arm discontinued therapy due to adverse events, with $6 \%$ in both groups experiencing at least one grade 4 toxicity. ${ }^{19}$ Consistent with these results, assessments undertaken during and after the study confirmed that there was no significant difference in health-related quality of life between the two arms, while patients with objective tumor responses experienced significant improvements on a variety of scales. ${ }^{50,51}$

An additional analysis of quality-adjusted time without symptoms of disease or toxicity of treatment (Q-TWiST) utilized the EGF100151 dataset. ${ }^{52}$ This scale subtracts all days between randomization and progression spent with grade 3/4 toxicities from the median PFS, resulting in an area under the curve representation of Q-TWiST (Fig. 1). Approximately one-third of patients in each arm experienced grade 3/4 toxicities prior to progression, with a mean duration of 2 weeks in both groups. However, the increased PFS in patients receiving lapatinib plus capecitabine conferred a significant TWiST advantage over those on capecitabine alone (32.1 vs 21.3 weeks, $P<0.0001) .{ }^{52}$ When all grades of toxicities were included, patients in the combination arm experienced an additional 4-5 weeks with adverse events, but they continued to enjoy a 6.1-week extension in Q-TWiST over the monotherapy group $(P=0.006)$. This advantage held true regardless of the relative importance that individual patients placed on delaying relapse or avoiding toxicity. ${ }^{52}$

In MBC patients with brain metastases at commencement of therapy, a phase II study suggested that there may be symptomatic neurological benefits associated with treatment..$^{53}$ In an extension arm of this study, 
Table 4 Potential drug interactions with lapatinib

\begin{tabular}{|c|c|c|c|}
\hline Class & Agents & Effects & Comments \\
\hline CYP3A4 inducers & & $\begin{array}{l}\text { May reduce lapatinib } \\
\text { concentration }\end{array}$ & $\begin{array}{l}\text { Consider dosage increase for } \\
\text { lapatinib and/or the interacting } \\
\text { agent when co-administration is } \\
\text { unavoidable }\end{array}$ \\
\hline Antibiotics & Rifabutin, rifampicin, rifapentine & & \\
\hline Anticonvulsants & $\begin{array}{l}\text { Carbamazepine, fosphenytoin, } \\
\text { phenobarbitone, phenytoin }\end{array}$ & & \\
\hline Antiretrovirals & Efavirenz, nevirapine & & \\
\hline Oral corticosteroids & $\begin{array}{l}\text { Cortisone, dexamethasone, } \\
\text { hydrocortisone, } \\
\text { methylprednisone, prednisone }\end{array}$ & & \\
\hline Other & $\begin{array}{l}\text { Hypericum perforatum (St John's } \\
\text { wort), irinotecan, modafinil }\end{array}$ & & $\begin{array}{l}\text { Patients should be advised not to } \\
\text { take St John's wort while on } \\
\text { lapatinib therapy }\end{array}$ \\
\hline CYP3A4 inhibitors & & $\begin{array}{l}\text { May increase lapatinib } \\
\text { concentration }\end{array}$ & $\begin{array}{l}\text { Consider dosage reduction for } \\
\text { lapatinib and/or the interacting } \\
\text { agent when co-administration is } \\
\text { unavoidable }\end{array}$ \\
\hline Antibiotics & Clarithromycin, erythromycin & & \\
\hline Antidepressants & Nefazodone, fluvoxamine & & \\
\hline Antifungals & $\begin{array}{l}\text { Fluconazole, itraconazole, } \\
\text { ketoconazole, voriconazole }\end{array}$ & & \\
\hline Antihypertensives & Verapamil, diltiazem & & \\
\hline Antiretrovirals & $\begin{array}{l}\text { Amprenavir, atazanavir, } \\
\text { delaviridine, fosamprenavir, } \\
\text { indinavir, lopinavir, nelfinavir, } \\
\text { ritonavir, saquinavir }\end{array}$ & & \\
\hline Other & $\begin{array}{l}\text { Amiodarone, aprepitant, } \\
\text { cimetidine, grapefruit (including } \\
\text { juice) }\end{array}$ & & $\begin{array}{l}\text { Patients should be advised not to } \\
\text { consume grapefruit or its juice } \\
\text { while on lapatinib therapy }\end{array}$ \\
\hline Antacids & $\begin{array}{l}\text { Preparations containing alginic } \\
\text { acid, aluminium hydroxide, } \\
\text { calcium carbonate, magnesium } \\
\text { hydroxide }\end{array}$ & $\begin{array}{l}\text { May reduce lapatinib } \\
\text { absorption. }\end{array}$ & $\begin{array}{l}\text { Avoid for up to } 1 \mathrm{~h} \text { before and } \\
\text { after lapatinib dosing }\end{array}$ \\
\hline Antidiarrheals & $\begin{array}{l}\text { Preparations containing atropine, } \\
\text { codeine, loperamide }\end{array}$ & $\begin{array}{l}\text { May increase lapatinib } \\
\text { absorption. }\end{array}$ & Discontinue once diarrhea resolves \\
\hline Herbal preparations & $\begin{array}{l}\text { Echinacea, evening primrose oil, } \\
\text { Ginkgo biloba, ginseng, grape } \\
\text { seed, kava, valerian }\end{array}$ & Various/unknown. & $\begin{array}{l}\text { Discourage concurrent use with } \\
\text { lapatinib where possible }\end{array}$ \\
\hline
\end{tabular}

Consult local product information for possible capecitabine interactions.

capecitabine plus lapatinib was associated with a 20\% reduction in the CNS tumor volume in $37 \%$ of patients. ${ }^{34}$ Post-marketing observational studies have also noted improvements in neurological signs and symptoms in at least $25 \%$ of MBC patients with brain metastases who received lapatinib plus capecitabine. ${ }^{33,54}$ While these preliminary CNS data await confirmation, clinicians should consider neurological criteria when assessing clinical benefit in this subgroup.

\section{MINIMIZING ADVERSE EVENTS}

Data from EGF100151 suggest that most patients receiving lapatinib plus capecitabine can expect to experience at least one adverse event, the most common being diarrhea, nausea, vomiting or dermatological effects. ${ }^{19,52}$ Subsequent clinical experience has not identified additional adverse events beyond those recorded in the pivotal trial. ${ }^{55}$ Nausea and vomiting can usually be 


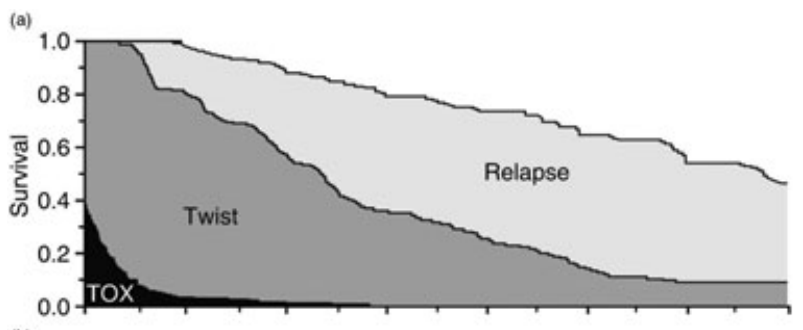

(b)

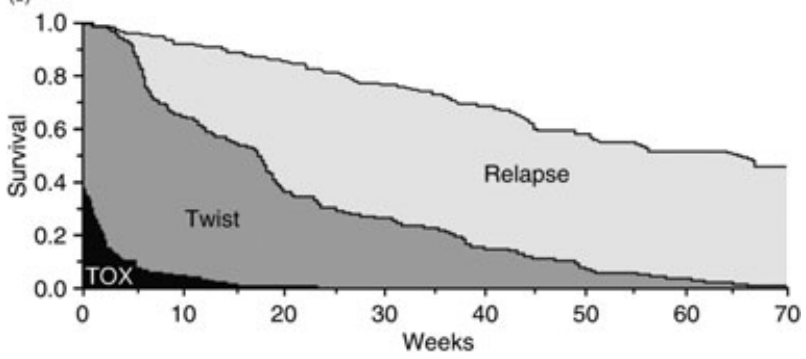

Figure 1 Partitioned survival curves representing Q-TWiST area under the curve for both (a) lapatinib plus capecitabine and (b) capecitabine monotherapy arms in the EGF100151 phase III trial where toxicity (TOX) equals AE, adverse events; ITT, intent-to-treat; TOX, toxicity; Twist, time without symptoms or toxicity. Reprinted by permission from Macmillan Publishers Ltd: Sherrill B et al. Br J Cancer 2008; 99: 711-15. ${ }^{52}$

managed via standard protocols, while diarrhea and skin events are generally the most bothersome to patients and may develop into serious toxicities. However, both can be minimized with clear patient education and a proactive management plan on commencing therapy. In particular, patients should understand what constitutes an unacceptable level of toxicity and be encouraged to report problems urgently. Routine monitoring may also detect incipient problems (Table 2). Aside from specific instances noted below, when the severity of an adverse event suggests that dosage adjustment is required, capecitabine should be altered before lapatinib dosing is revised. However, if toxicity necessitates the permanent withdrawal of one agent, the entire regimen should be ceased.

\section{Dermatological events}

Dermatological adverse events are frequent with lapatinib therapy, although Caucasians appear more sensitive than individuals of Asian ethnicity. ${ }^{43,56}$ While skin dryness is sometimes observed during trastuzumab treatment - suggesting that HER2 inhibition may play a role-EGFR inhibition is the major contributor to cutaneous events such as rash, xerosis and paronychia. ${ }^{56}$ EGFR blockade is associated with reduced growth and migration of keratinocytes, which may manifest as inflammation, epidermal thinning or superinfection. Such events are particularly prominent at sites of cutaneous injury - including minor trauma or established lesions such as solar keratoses - and may induce an escalating cycle of inflammation and epidermal damage. The likelihood of photosensitivity or palmar plantar erythrodysesthesia (hand-foot syndrome) is substantially increased by concurrent administration of capecitabine. ${ }^{37,56}$ The resultant cosmetic and physical effects may have marked psychological sequelae, leading to compromised adherence. ${ }^{56}$ Consultation with a dermatologist should therefore be considered when troublesome skin reactions affect patients who are experiencing a clinical benefit with lapatinib plus capecitabine.

The most common dermatologic adverse event associated with this regimen is palmar plantar erythrodysesthesia, which affects approximately $50 \%$ of patients (Fig. 2a). ${ }^{56}$ This is largely a consequence of capecitabine treatment but may be exacerbated by lapatinib, heat and trauma. The syndrome may appear at any time during treatment and persists for a median of 3 weeks. ${ }^{56,57}$ Initial management consists of reducing and/or delaying subsequent doses of capecitabine, which is mandatory if the severity reaches grade 3 or higher. Although there are no clear evidence-based strategies for management, emollient creams are recommended both for prophylaxis and treatment, while dietary pyridoxine supplementation may provide symptomatic benefits. ${ }^{57}$

A papulopustular rash is also common among patients taking lapatinib, usually appearing early and affecting areas of skin exposed to sunlight (Fig. 2b). Unlike other EGFR-targeted therapies such as cetuximab, there is no apparent association between skin rash and the efficacy of lapatinib. ${ }^{43}$ Where possible, patients should be encouraged to persevere with lapatinib through an episode, as the rash is generally mild and short-lived (median duration 1 month). ${ }^{56}$ Although sometimes described as acneform, this presentation is dermatologically distinct from acne vulgaris and should not be managed with retinoids. ${ }^{43}$ Rather, patients should be prescribed topical $0.5 \%$ hydrocortisone, or more potent corticosteroids in severe cases. Suspected superinfection should be treated with oral tetracycline antibiotics such as minocycline or doxycycline.

Xerosis results from decreases in natural oil production and water retention. It may manifest as rough, itchy patches or as small fissures or 'paper cuts', particularly on the hands (Fig. 2c). This condition may seem minor but it is particularly troubling for affected individuals. The sensitivity of skin during treatment means that all 

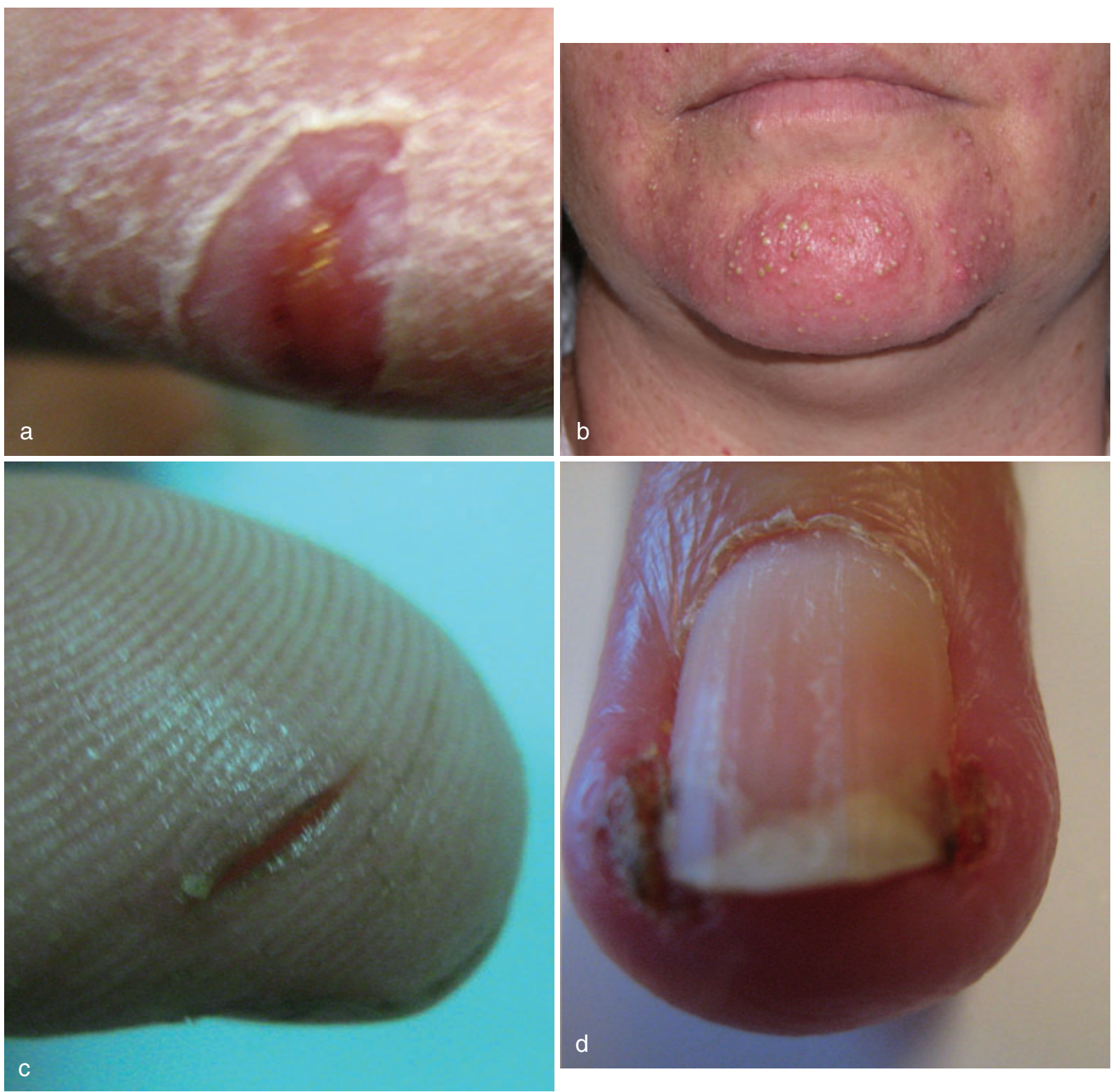

Figure 2 Typical presentations of dermatological adverse events that may occur during lapatinib plus capecitabine therapy: (a) palmar plantar erythrodysesthesia; (b) papulopustular rash; (c) xerosis; (d) paronychia. All images courtesy of Dr Fran Boyle.

patients should be advised to minimize cutaneous trauma (for instance, by wearing gloves when cleaning or gardening), to use soap substitutes when washing and to avoid alcohol-based skin lotions. Regular prophylactic application of non-perfumed, hypo-allergenic hand creams may also be beneficial; lanolin is not recommended but suitable choices include vitamin $\mathrm{E}$, petroleum jelly or commercial emollient preparations.
Troublesome pruritus can be managed with nonsedating oral antihistamines such as loratadine or desloratadine.

Paronychia (Fig. 2d) tends to emerge later in treatment and frequently presents with Staphylococcus aureus superinfection. This painful condition may be exacerbated by trauma, reinforcing the need for skin protection. Treatments include soaking the affected nail 
bed in a 1:1 solution of vinegar and water, or with the application of a silver nitrate 'caustic pencil' to granulation tissue. Prescription of antibiotics with S. aureus coverage - such as topical mupirocin cream or oral agents such as cefaclor or clindamycin - may also be appropriate.

\section{Gastrointestinal events}

Most patients receiving lapatinib plus capecitabine are likely to experience diarrhea, necessitating a proactive approach to its management. ${ }^{43,55,58}$ The mechanism underlying lapatinib-induced diarrhea is unclear but it appears to be a class effect of EGFR inhibitors, mediated either via local activity in the gastric epithelium or by systemic exposure, and exacerbated by the concurrent administration of capecitabine. ${ }^{58}$ In regions where communicable gastrointestinal diseases are prevalent, the possibility of intercurrent infection should also be considered.

Clinical trial data suggest that grade $1 / 2$ diarrhea occurs in approximately $50 \%$ of patients receiving lapatinib plus capecitabine. A further $13 \%$ experience grade 3 and $1 \%$ grade 4 toxicity, although no treatment-related diarrheal fatalities were reported in EGF100151. ${ }^{58}$ Nearly half of all cases emerge within the first week of treatment, with a median duration of 4-5 days for grade $1 / 2$ diarrhea and 8 days for more severe events. Data from lapatinib monotherapy studies suggest that although it is unusual for grade $1 / 2$ presentations to worsen in severity, patients over 70 years of age may be at higher risk of severe diarrhea. ${ }^{58}$

Management of diarrhea is generally straightforward, provided that patients are educated in advance and treatment is instigated early. EGF100151 investigators were required to follow the American Society of Clinical Oncology guidelines for the management of treatmentinduced diarrhea. ${ }^{59}$ These suggest outpatient management of grade $1 / 2$ events with loperamide and dietary modification such as reduced fiber intake, avoidance of lactose and increased oral fluids. At the clinician's discretion, patients may instigate therapy themselves but they must be told to inform the oncology team when diarrhea develops. For grade 2 events, both lapatinib and capecitabine should be withheld until symptoms resolve; for more persistent diarrhea, loperamide should be replaced with octreotide plus oral antibiotics if indicated. Grade $\geq 3$ events or complicated grade 1/2 diarrhea may require inpatient treatment with intravenous hydration, octreotide therapy and dietary measures. Suspension of both lapatinib and capecitabine is indicated for up to 14 days, while oral or intravenous antibiotics should be administered as needed. ${ }^{58}$ In most cases diarrhea resolves with appropriate management, so the permanent discontinuation of lapatinib and capecitabine is only occasionally necessary. If capecitabine or lapatinib (or both) are temporarily withheld, treatment should recommence at lower doses as noted in the local product information. ${ }^{37,60}$

\section{Cardiac and pulmonary events}

The risk of cardiotoxicity with lapatinib appears lower than was inferred from clinical experience with trastuzumab. ${ }^{40,43}$ A recent analysis of 3689 patients treated with lapatinib for solid tumors found that symptomatic congestive heart failure (CHF) affected $0.2 \%$ while asymptomatic events (predominantly LVEF decrease) occurred in a further $1.4 \%$ - frequencies similar to those in the control group. ${ }^{38}$ Similar rates of symptomatic $(0.5 \%)$ and asymptomatic $(2.0 \%)$ LVEF decline were reported for patients treated with lapatinib plus capecitabine in EGF100151, and subsequently corroborated by post-marketing surveillance. ${ }^{19,55}$ LVEF decline usually occurs within the first 3 months of therapy and tends to be relatively mild; both the incidence and severity appear unrelated to prior exposure to anthracyclines or trastuzumab. ${ }^{38}$

Unlike anthracycline-induced cardiotoxicity, the myocardial effects of lapatinib are apparently reversible..$^{38,43}$ LVEF generally returns to baseline values after the cessation of lapatinib, but recovery has also been reported despite continuing treatment. ${ }^{19,38}$ Nevertheless, only patients with adequate cardiac function should commence lapatinib and oncologists are urged to follow recommended cardiac monitoring protocols (Table 2). As there are no firm data regarding the management of asymptomatic LVEF decline, clinicians may wish to utilize the relevant trastuzumab recommendations, which suggest monthly monitoring if LVEF stabilizes at $\geq 40 \% .{ }^{43,61}$ Lapatinib should be withheld and cardiac evaluation undertaken again in 1 month if the patient experiences symptomatic CHF, if there is an absolute LVEF fall $\geq 20 \%$, or if LVEF falls below local limits (generally $<40 \%$ ). ${ }^{43,61}$ Discussion of the relative risks and benefits of reinstituting therapy can then commence with the patient and a cardiologist.

Although potentially serious, interstitial lung disease and pneumonitis were noted neither in EGF100151 nor in phase I/II trials of lapatinib monotherapy. ${ }^{15,17,19}$ Four instances were, however, reported among 265 patients in a European early access program $(1.5 \%) .{ }^{55}$ Apart from regular clinical review for symptoms such as dyspnea, dry cough or chest pain, no specific monitoring 
is required. However, if pulmonary effects reach grade $\geq 3$ severity, treatment should be ceased. ${ }^{37}$

\section{Hepatic and renal effects}

In common with other tyrosine kinase inhibitors, lapatinib may alter liver function. Grade 4 hyperbilirubinemia was reported in approximately $1-2 \%$ of patients in a phase II study of lapatinib monotherapy for HER2positive or HER2-negative MBC. ${ }^{15}$ One treatmentrelated fatality $(0.4 \%)$ has been attributed to hepatic dysfunction during a clinical experience program of lapatinib plus capecitabine for MBC. ${ }^{55}$ Because disease progression commonly deranges liver function in $\mathrm{MBC}$ patients, other causes should be excluded prior to amending lapatinib or capecitabine dosing. Unless progression is confirmed, a rise in ALT/AST or bilirubin above local limits would normally require the cessation of lapatinib. The oncologist may consider restarting treatment if hepatic function subsequently normalizes.

Lapatinib exhibits minimal renal excretion and has no demonstrated propensity to cause nephrotoxicity. ${ }^{37}$ However, compromised renal clearance is associated with increased capecitabine toxicity, necessitating revised dosing according to the local product information. ${ }^{60}$

\section{DISCUSSION}

The introduction of lapatinib into clinical practice allows oncologists to maintain HER2-targeted MBC therapy following the development of trastuzumab resistance. The recommendations in this article can assist clinicians in maximizing symptomatic and quality of life benefits associated with lapatinib plus capecitabine, while minimizing the potential and severity of adverse events. Although the regimen is generally well tolerated by patients, as an oral outpatient therapy with potential for serious adverse events, a proactive management plan and a commitment to patient education are essential to optimal outcomes.

A review of ongoing clinical trials of lapatinib is beyond the scope of this article, but it is worthwhile noting current areas of investigation. These include lapatinib monotherapy for MBC; combinations of lapatinib with cytotoxic agents, targeted therapies or radiation for breast cancer and other solid tumors; lapatinib plus capecitabine for the treatment of brain metastases in MBC patients; and ongoing pharmacogenomic and tumor biomarker analyses. Many of these investigations address areas of pressing clinical need and their results will be of considerable interest to oncologists across the globe.

\section{ACKNOWLEDGMENTS}

The authors are all members of the Australian GlaxoSmithKline Breast Cancer Advisory Panel. Each author has also served as an investigator in GlaxoSmithKlinesponsored clinical trials of lapatinib, some of which are ongoing at the time of publication. The content for this article was presented, discussed and agreed by the authors at an advisory board meeting supported by GlaxoSmithKline. A freelance medical writer, Peter Hobbins, undertook the writing of the first draft and coordinated author amendments. Each author contributed to reviewing and revising the manuscript until group consensus on the content was achieved. While GlaxoSmithKline was invited to review the article prior to submission to ensure the accuracy of technical and regulatory information, the authors take full responsibility for the content and expression of the submitted manuscript.

\section{REFERENCES}

1 Slamon DJ, Leyland-Jones B, Shak S et al. Use of chemotherapy plus a monoclonal antibody against HER2 for metastatic breast cancer that overexpresses HER2. N Engl J Med 2001; 344: 783-92.

2 Marty M, Cognetti F, Maraninchi D et al. Randomized phase II trial of the efficacy and safety of trastuzumab combined with docetaxel in patients with human epidermal growth factor receptor 2-positive metastatic breast cancer administered as first-line treatment: the M77001 study group. J Clin Oncol 2005; 23: 4265-74.

3 Slamon D, Eiermann W, Robert N et al. BCIRG 006: 2nd interim analysis phase III randomized trial comparing doxorubicin and cyclophosphamide followed by docetaxel (ACT) with doxorubicin and cyclophosphamide followed by docetaxel and trastuzumab (ACTH) with docetaxel, carboplatin and trastuzumab (TCH) in Her2neu positive early breast cancer patients. Breast Cancer Res Treat 2006; 100: (Suppl. 1): Abstract 52.

4 Vogel CL, Cobleigh MA, Tripathy D et al. Efficacy and safety of trastuzumab as a single agent in first-line treatment of HER2-overexpressing metastatic breast cancer. J Clin Oncol 2002; 20: 719-26.

5 Nahta R, Yu D, Hung M-C, Hortobagyi GN, Esteva FJ. Mechanisms of disease: understanding resistance to HER2targeted therapy in human breast cancer. Nat Clin Pract Oncol 2006; 3: 269-80. 
6 Nahta R, Esteva FJHER2 therapy: molecular mechanisms of trastuzumab resistance. Breast Cancer Res 2006; 8: 215-23.

7 Manoir JM, Francia G, Man S et al. Strategies for delaying or treating in vivo acquired resistance to trastuzumab in human breast cancer xenografts. Clin Cancer Res 2006; 12: 904-16.

8 Valabrega G, Montemurro F, Aglietta M. Trastuzumab: mechanism of action, resistance and future perspectives in HER2-overexpressing breast cancer. Ann Oncol 2007; 18: 977-84.

9 Xia W, Liu LH, Ho P, Spector NL. Truncated ErbB2 receptor $(\mathrm{p} 95 \mathrm{ErbB} 2)$ is regulated by heregulin through heterodimer formation with ErbB3 yet remains sensitive to the dual EGFR/ErbB2 kinase inhibitor GW572016. Oncogene 2004; 23: 646-53.

10 Nahta R, Yuan LXH, Du Y, Esteva FJ. Lapatinib induces apoptosis in trastuzumab-resistant breast cancer cells: effects on insulin-like growth factor I signaling. Mol Cancer Ther 2007; 6: 667-74.

11 Rusnak DW, Alligood KJ, Mullin RJ et al. Assessment of epidermal growth factor receptor (EGFR, ErbB1) and HER2 (ErbB2) protein expression levels and response to lapatinib (Tykerb, GW572016) in an expanded panel of human normal and tumor cell lines. Cell Prolif 2007; 40: 580-94.

12 Konecny GE, Pegram MD, Venkatesan N et al. Activity of the dual kinase inhibitor lapatinib (GW572016) against HER-2-overexpressing and trastuzumab-treated breast cancer cells. Cancer Res 2006; 66: 1630-39.

13 Rodriguez AA, Chang J, Li X et al. Decrease in tumorigenic breast cancer cells with neoadjuvant lapatinib. Eur J Cancer Suppl 2008; 6: 108 (Abstract 204).

14 Gomez HL, Chavez MA, Doval DC et al. A phase II, randomized trial using the small molecule tyrosine kinase inhibitor lapatinib as a first-line treatment in patients with FISH positive advanced or metastatic breast cancer. J Clin Oncol 2005; 23 (Suppl. 16): 203s (Abstract 3046).

15 Burstein HJ, Storniolo AM, Franco S et al. A phase II study of lapatinib monotherapy in chemotherapyrefractory HER2-positive and HER2-negative advanced or metastatic breast cancer. Ann Oncol 2008; 19: 106874.

16 Schwartz G, Chu QS-C, Hammond LA et al. Phase I clinical, biology \& pharmacokinetic study of the combination of GW 572016 and capecitabine in patients with advanced solid tumors. J Clin Oncol 2004; 22 (Suppl. 14):212s (Abstract 3070).

17 Burris HA III, Hurwitz HI, Dees EC et al. Phase I safety, pharmacokinetics, and clinical activity study of lapatinib (GW572016), a reversible dual inhibitor of epidermal growth factor receptor tyrosine kinases, in heavily pretreated patients with metastatic carcinomas. J Clin Oncol 2005; 23: 5305-13.
18 Geyer CE, Forster J, Lindquist D et al. Lapatinib plus capecitabine for HER2-positive advanced breast cancer. N Engl J Med 2006; 355: 2733-43.

19 Cameron D, Casey M, Press M et al. A phase III randomized comparison of lapatinib plus capecitabine versus capecitabine alone in women with advanced breast cancer that has progressed on trastuzumab: updated efficacy and biomarker analyses. Breast Cancer Res Treat 2008; 112: 533-43 [Epub ahead of print].

20 Office of Oncology Drug Products. FDA Approves Tykerb (Lapatinib Tablets) for Advanced Metastatic Breast Cancer Patients. US Food and Drug Administration Center for Drug Evaluation and Research, Washington DC 2007. [Updated 16 March 2007; Cited 2 September 2008]. Available from: http://www.fda.gov/cder/offices/oodp/ whatsnew/lapatinib.htm

21 Australian Drug Evaluation Committee. ADEC 252nd Meeting Recommendations, Resolution 9073. Australian Government Department of Health and Ageing, Therapeutic Goods Administration, Canberra 2007. [Updated 11 July 2007; Cited 2 September 2008]. Available from: http:// www.tga.health.gov.au/docs/html/adec/adec0252.htm

22 Pharmaceutical Benefits Scheme. Pharmaceutical Benefits Schedule for Lapatinib. Australian Government Department of Health and Ageing, Canberra 2008. [Updated 1 September 2008; Cited 2 September 2008]. Available from: http://www.pbs.gov.au/html/healthpro/search/ results?term=lapatinib\&scope=PBS+STATIC\&formtype $=$ simple

23 European Medicines Agency. EPARs for Authorised Medicinal Products for Human Use: Tyverb (H-C-795). European Medicines Agency, London 2008. [updated 10 June 2008; cited 2 September 2008]. Available from: http:// www.emea.europa.eu/humandocs/Humans/EPAR/tyverb/ tyverb.htm

24 National Comprehensive Cancer Network. NCCN Clinical Practice Guidelines in Oncology: Breast Cancer v.2.2008. National Comprehensive Cancer Network, Fort Washington, PA 2008. [Cited 2 September 2008]. Available from: http://www.nccn.org/professionals/physician_gls/PDF/ breast.pdf

25 Toi M, Iwata H, Fujiwara Y et al. Biomarker analyses in Japanese refractory advanced breast cancer patients treated with lapatinib monotherapy. Presented at the 30th Annual San Antonio Breast Cancer Symposium, San Antonio, USA, 2007 (Abstract 5114).

26 Solomayer EF, Becker S, Pergola-Becker G et al. Comparison of HER2 status between primary tumor and disseminated tumor cells in primary breast cancer patients. Breast Cancer Res Treat 2006; 98: 179-84.

27 Fehm T, Becker S, Duerr-Stoerzer S et al. Determination of HER2 status using both serum HER2 levels and circulating tumor cells in patients with recurrent breast cancer whose primary tumor was HER2 negative or of unknown HER2 status. Breast Cancer Res 2007; 9: R74. 
28 Wolff AC, Hammond ME, Schwartz JN et al. American Society of Clinical Oncology/College of American Pathologists guideline recommendations for human epidermal growth factor receptor 2 testing in breast cancer. Arch Pathol Lab Med 2007; 131: 1-28.

29 Greshock J, Cheng J, Rusnak D et al. Genome-wide DNA copy number predictors of lapatinib sensitivity in tumorderived cell lines. Mol Cancer Ther 2008; 7: 935-43.

30 Kirsch DG, Hochberg FH. Targeting HER2 in brain metastases from breast cancer. Clin Cancer Res 2003; 9: 5435-36.

31 Montagna E, Cancello G, D'Agostino D et al. Central nervous system metastases in a cohort of metastatic breast cancer patients treated with trastuzumab. Cancer Chemother Pharmacol 2008; 63: 275 [Epub ahead of print].

32 Gril B, Palmieri D, Bronder JL et al. Effect of lapatinib on the outgrowth of metastatic breast cancer cells to the brain. J Natl Cancer Inst 2008; 100: 1092-103.

33 Boccardo F, Kaufman B, Baselga J et al. Evaluation of lapatinib (Lap) plus capecitabine (Cap) in patients with brain metastases (BM) from HER2+ breast cancer (BC) enrolled in the Lapatinib Expanded Access Program (LEAP) and French Authorisation Temporaire d'Utilisation (ATU). J Clin Oncol 2008; 26 (Suppl. 15): 64s (Abstract 1094).

34 Lin NU, Paul D, Dieras V et al. Lapatinib and capecitabine for the treatment of brain metastases in patients with HER2+ breast cancer: an updated analysis from EGF105084. Presented at the 30th Annual San Antonio Breast Cancer Symposium, San Antonio, USA, 2007 (Abstract 6076).

35 Lin NU. EGF105084, a phase II study of lapatinib for brain metastases in patients (pts) with HER2+ breast cancer following trastuzumab $(\mathrm{H})$ based systemic therapy and cranial radiotherapy (RT). J Clin Oncol 2007; 25 (Suppl. 18): 35s (Abstract 1012).

36 Lin NU, Carey LA, Liu MC et al. Phase II trial of lapatinib for brain metastases in patients with human epidermal growth factor receptor 2-positive breast cancer. J Clin Oncol 2008; 26: 1993-99.

37 GlaxoSmithKline. Tykerb (lapatinib ditosylate) Australian Approved Product Information (Issue 2), 2007.

38 Perez EA, Koehler M, Byrne J, Preston AJ, Rappold E, Ewer MS. Cardiac safety of lapatinib: pooled analysis of 3689 patients enrolled in clinical trials. Mayo Clin Proc 2008; 83: 679-86.

39 Perez EA. Cardiac toxicity of ErbB2-targeted therapies: what do we know? Clin Breast Cancer 2008; 8: S114-20.

40 Force T, Krause DS, Van Etten RA. Molecular mechanisms of cardiotoxicity of tyrosine kinase inhibition. Nat Rev Cancer 2007; 7: 332-44.

41 Winkeljohn DL. Oral chemotherapy medications: the need for a nurse's touch. Clin J Oncol Nurs 2007; 11: 79396.
42 Kirk M, Hudis C. Insight into barriers against optimal adherence to oral hormonal therapy in women with breast cancer. Clin Breast Cancer 2008; 8: 155-61.

43 Moy B, Goss PE. Lapatinib-associated toxicity and practical management recommendations. Oncologist 2007; 12: 756-65.

44 MIMS. eMIMS DrugAlert (lapatinib). MIMS, Sydney 2008.

45 Koch KM, Beelen AP, Ho PTC, Roychowdhury DF. The value of label recommendations: how to dose lapatinib. J Clin Oncol 2007; 25: 5331-32.

46 Ratain MJ, Cohen EE. The value meal: how to save $\$ 1,700$ per month or more on lapatinib. J Clin Oncol 2007; 25: 3397-98.

47 Messori A. Effect of food on lapatinib pharmacokinetics. J Clin Oncol 2007; 25: 5332-33.

48 Rahman A, Pazdur R, Wang Y, Huang S-M, Lesko L. The value meal: effect of food on lapatinib bioavailability. J Clin Oncol 2007; 25: 5333-34.

49 Trippoli S. Controversies in using lapatinib at reduced dosage with food. J Clin Oncol 2007; 25: 5333.

50 Zhou X, Segreti A, Cameron D et al. Effect of lapatinib plus capecitabine $(\mathrm{L}+\mathrm{C})$ on quality of life $(\mathrm{QOL})$ compared to capecitabine $(\mathrm{C})$ alone in ErbB2+ metastatic breast cancer (MBC): an exploratory analysis. [ ] J Clin Oncol 2008; 26 (Suppl. 15): 509s (Abstract 9532).

51 Zhou X, Segreti A, Cella D et al. Lapatinib plus capecitabine versus capecitabine alone for ErbB2-positive metastatic breast cancer (MBC) - quality of life (QOL) assessment. Eur J Cancer Suppl 2008; 6: 216-17 (Abstract 565).

52 Sherrill B, Amonkar MM, Stein S, Walker M, Geyer C, Cameron D. Q-TWiST analysis of lapatinib combined with capecitabine for the treatment of metastatic breast cancer. Br J Cancer 2008; 99: 711-15.

53 Lin NU, Roché HH, Dieras V et al. A physician-reported neurological signs and symptoms worksheet (NSS WS) in EGF105084: a phase II study of lapatinib (lap) for pts with recurrent brain metastases (BM) from HER2+ breast cancer (BC). J Clin Oncol 2008; 26 (Suppl. 15): 60s (Abstract 1078)

54 Kaufman B, Weitzen R, Goldfarb A et al. A singleinstitution experience from the Lapatinib Expanded Access Program - effect of lapatinib and capecitabine combination therapy on CNS metastases in patients with ErbB2+ metastatic breast cancer. Eur J Cancer Suppl 2008; 6: 175 (Abstract 419).

55 De Placido S, Link J, Conte PF et al. Lapatinib Expanded Access Program (LEAP): design, operation and initial safety data. Breast Cancer Research and Treatment, 2007: 106 (Suppl 1), (Abstract 6077).

56 Lacouture ME, Laabs SM, Koehler M et al. Analysis of dermatologic events in patients with cancer treated with lapatinib. Breast Cancer Res Treat 2008; [Epub ahead of print]. 
57 Gressett SM, Stanford BL, Hardwicke F. Management of hand-foot syndrome induced by capecitabine. J Oncol Pharm Pract 2006; 12: 131-41.

58 Crown JP, Burris HA, Boyle F et al. Pooled analysis of diarrhea events in patients with cancer treated with lapatinib. Breast Cancer Res Treat 2008; 112: 317-25.

59 Benson AB III, Ajani JA, Catalano RB et al. Recommended guidelines for the treatment of cancer treatment-induced diarrhea. J Clin Oncol 2004; 22: 2918-26.
60 Roche Products. Xeloda (capecitabine) Australian Approved Product Information, 2008.

61 Ewer MS, Perez EA, Baselga J et al. Cardiac safety guidelines for the adjuvant use of trastuzumab (Herceptin $\left.{ }^{\circledR}\right)$ in HER2-positive early breast cancer. Breast 2007; 16: S63 (Abstract P176).

\section{APPENDIX I}

A patient diary or medication calendar can assist in ensuring optimal dosing and adherence to oral lapatinib plus capecitabine regimens. This Australian example is reproduced with the kind permission of GlaxoSmithKline.

\section{Tykerb and Xeloda diary}

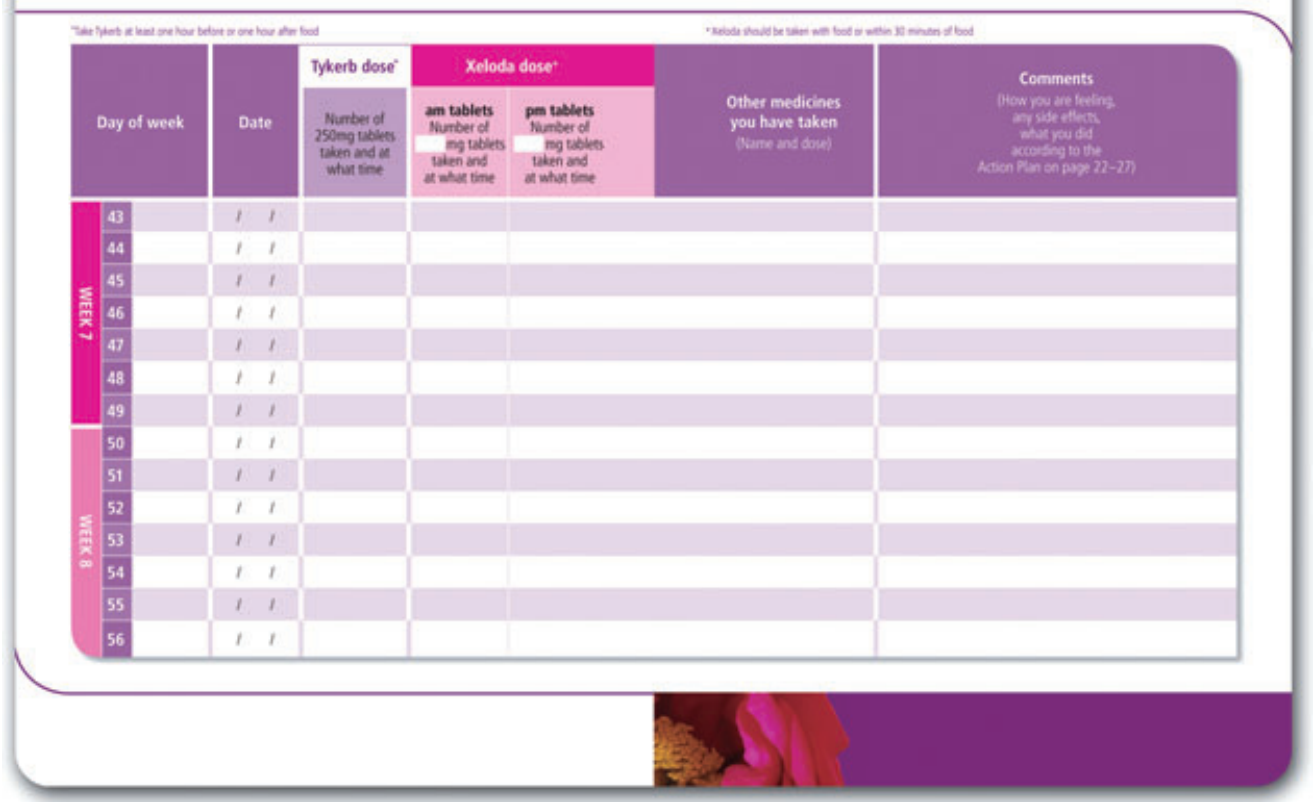

